\title{
NEUROBLASTOMA NECK IN A 7-YEAR-OLD GIRL: A VERY RARE PRESENTATION
}

\author{
RAMEEZ SHAH ${ }^{1}$, SK NURUL FATAH RUMI²
}

\begin{abstract}
:
Neuroblastoma in neck is a very rare presentation. The age range of neuroblastoma is broad, but only $10 \%$ of cases are reported in people older than 5years of age, and is most commonly diagnosed in children before age of 5years (with highest incidence in the first year of life), being slightly more common in boys. The girl child reported in our case, being mere 7 years old, presented with bilateral hard neck swellings, was not clinically suspected of this rare presentation, until Immunohistochemistry confirmed the diagnosis. This report underscores the need for prompt evaluation of patients (especially children) with common symptoms and uncommon presentation of the disease entity.
\end{abstract}

Key words: Neuroblastoma, Neck, Immunohistochemistry.

\section{Introduction:}

Neuroblastoma is the most common extra cranial solid cancer in childhood and the most common cancer in infancy, with an annual incidence of about six hundred and fifty cases per year in the U.S., ${ }^{1}$ and a hundred cases per year in the U.K. [2] Nearly half of neuroblastoma cases occur in children younger than two years. ${ }^{3}$ It is a neuroendocrine tumor, arising from any neural crest element of the sympathetic nervous system (S.N.S.). It most frequently originates in one of the adrenal glands, but can also develop in nerve tissues in the neck, chest, abdomen, or pelvis. The most common location for neuroblastoma to originate (i.e. the primary tumor) is on the adrenal glands.

1. MS (Thesis Part), Dept of ENT \& Head Neck Surgery, Dhaka Medical College Hospital, Dhaka Bangladesh.

2. Asst. Professor, Dept of ENT \& Head Neck Surgery, Dhaka Medical College Hospital, Dhaka Bangladesh.

Correspondence to : Dr. Rameez Shah (Srinagar, Kashmir), M.S. (Thesis Part), Dept of ENT \& Head Neck Surgery, Dhaka Medical College Hospital, Dhaka Bangladesh. Email: rameezshah527@yahoo.com
It is a neuroendocrine tumor, arising from any neural crest element of the sympathetic nervous system (S.N.S.). It most frequently originates in one of the adrenal glands, but can also develop in nerve tissues in the neck, chest, abdomen, or pelvis. The most common location for neuroblastoma to originate (i.e. the primary tumor) is on the adrenal glands. This occurs in $40 \%$ of localized tumors and in $60 \%$ of cases of widespread disease. Neuroblastoma can also develop anywhere along the sympathetic nervous system chain from the neck to the pelvis. Frequencies in different locations include: neck (1\%), chest (19\%), abdomen ( $30 \%$ non-adrenal), or pelvis $(1 \%)$. In rare cases, no primary tumor can be discerned. ${ }^{8}$

Rare but characteristic presentations include transverse myelopathy (tumor spinal cord compression, $5 \%$ of cases), treatment-resistant diarrhea (tumorvasoactive intestinal peptide secretion, $4 \%$ of cases), Horner's syndrome (cervical tumor, $2.4 \%$ of cases), opsoclonus myoclonus syndrome ${ }^{[9]}$ and ataxia(suspected paraneoplastic cause, $1.3 \%$ of cases), and hypertension (catecholamine secretion or renal artery compression, $1.3 \%$ of cases). ${ }^{10}$

Neuroblastoma often spreads to other parts of the body before any symptoms are apparent and 50 to $60 \%$ of all neuroblastoma cases present with metastases. ${ }^{10}$

Neuroblastoma is one of the few human malignancies known to demonstrate spontaneous regression from an undifferentiated state to a completely benign cellular appearance. ${ }^{4}$ It is a disease exhibiting extreme heterogeneity, and is stratified into three risk 
categories: low, intermediate, and high risk. Low-risk disease is most common in infants and good outcomes are common with observation only or surgery, whereas high-risk disease is difficult to treat successfully even with the most intensive multi-modal therapies available. ${ }^{5}$

Neuroblastoma comprises $6-10 \%$ of all childhood cancers, and $15 \%$ of cancer deaths in children. The annual mortality rate is 10 per million children in the 0 - to 4-year-old age group, and 4 per million in the 4- to 9-year old age group. ${ }^{22}$

The highest incidence is in the first year of life, and some cases are congenital. The age range is broad, including older children and adults, ${ }^{[23]}$ but only $10 \%$ of cases occur in people older than 5 years of age. ${ }^{[12]}$

The first symptoms of neuroblastoma are often vague making diagnosis difficult. Fatigue, loss of appetite, fever, and joint pain are common. Symptoms depend on primary tumor locations and metastases if present: ${ }^{6}$

The etiology of neuroblastoma is not well understood. Certain cases however do run in families and have been linked to genetics. Familial neuroblastoma is caused by a very rare germline mutation in the anaplastic lymphoma kinase (ALK) gene. ${ }^{11}$

The diagnosis is usually confirmed by a surgical pathologist, taking into account the clinical presentation, microscopic findings, and other laboratory tests, especially Immunohistochemistrybeing a valuable and key tool in diagnosis. Neuroblastomas are characteristically positive for neuron specific enolase (NSE), and chromogranin A. Another sensitive and specific test for neuroblastoma is reverse transcriptase PCR (RTPCR) which can detect even small numbers of neuroblastoma cells in peripheral blood and bone marrow.
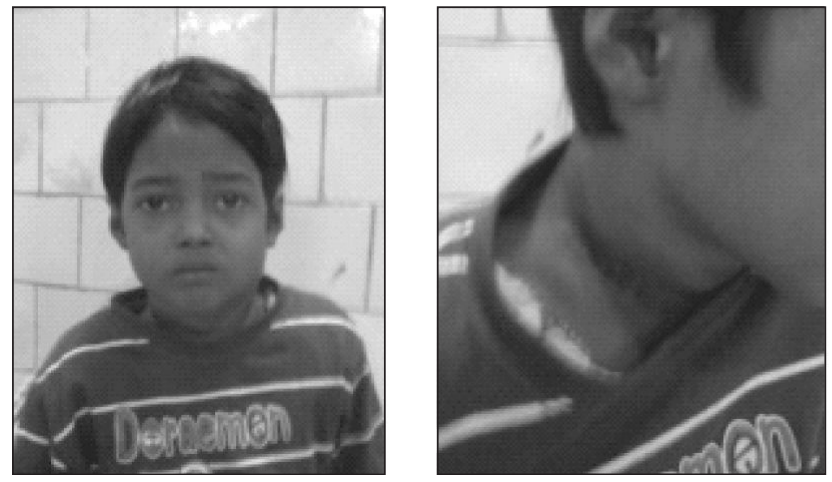

When the lesion is localized, it is generally curable. However, long-term survival for children with advanced disease older than 18 months of age is poor despite aggressive multimodal therapy (intensive chemotherapy, surgery, radiation therapy, stem cell transplant, differentiation agent isotretinoin also called 13-cis-retinoic acid, and frequently immunotherapy ${ }^{14}$ with anti-GD2 monoclonal antibody therapy).

The therapies for these different risk categories are very different.

Low-risk disease can frequently be observed without any treatment at all or cured with surgery alone. ${ }^{[13]}$ Intermediate-risk disease is treated with surgery and chemotherapy. ${ }^{[15]}$ High-risk neuroblastoma is treated with intensive chemotherapy, surgery, radiation therapy, bone marrow / hematopoietic stem cell transplantation, ${ }^{[16]}$ biological-based therapy with 13-cis-retinoic acid (isotretinoin or Accutane) $)^{[17]}$ and antibody therapy usually administered with the cytokines GM-CSFand IL-2. ${ }^{[18}$

Between $20 \%$ and $50 \%$ of high-risk cases do not respond adequately to induction high-dose chemotherapy and are progressive or refractory. ${ }^{19,20}$ Relapse after completion of frontline therapy is also common. Further treatment is available in phase I and phase II clinical trials that test new agents and combinations of agents against neuroblastoma, but the outcome remains very poor for relapsed high-risk disease. ${ }^{[21]}$

\section{Case Report:}

A 7 years old girl was admitted to the Dept of ENT \& Head Neck Surgery, Dhaka Medical College Hospital with bilateral, non-tender, hard mass in the neck for 3 months with evening rise of temperature, without any sweating or chills. She had no complaints of pain, no bleeding, no wt. loss, no fatigue, no itching and her appetite was good with normal bowel bladder habits.
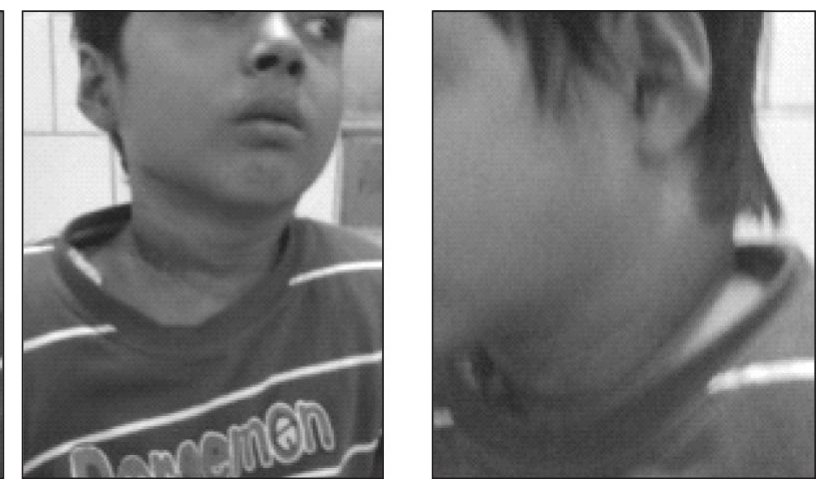

Figure 
The girl had no significant history of past illness, there was no contributory family history and she is well immunized.

The local examination of her neck revealed a $5 \mathrm{~cm} \times 4 \mathrm{~cm}$ hard mass on left side of her neck, extending from ramus of mandible upto above clavicle, $\&$ a $3 \mathrm{~cm} \times 2 \mathrm{~cm}$ hard mass on right side of her neck in the lower part of sternocleidomastoid muscle. The bilateral masses were smooth, irregular, non-tender, with raised temperature, hard, fixed with underlying structures \& free overlying skin. Neck movement was restricted on both sides. No systemic abnormality was detected. Her CBC revealed neutrophilic leucocytosis with thrombocytosis (ESR was not raised), $\mathrm{CXR}(\mathrm{P} / \mathrm{A}$ ) revealed pulmonary inflammatory lesion, Renal function was with normal range, USG of neck revealed extensive cervical lymphadenopathy, USG(W/A) showed mild ascites with bilateral enlarged ovaries. FNAC revealed no granuloma but evidence of metastatic deposits. Open biopsy was performed \& the histopathology report suggested malignant round cell tumor.

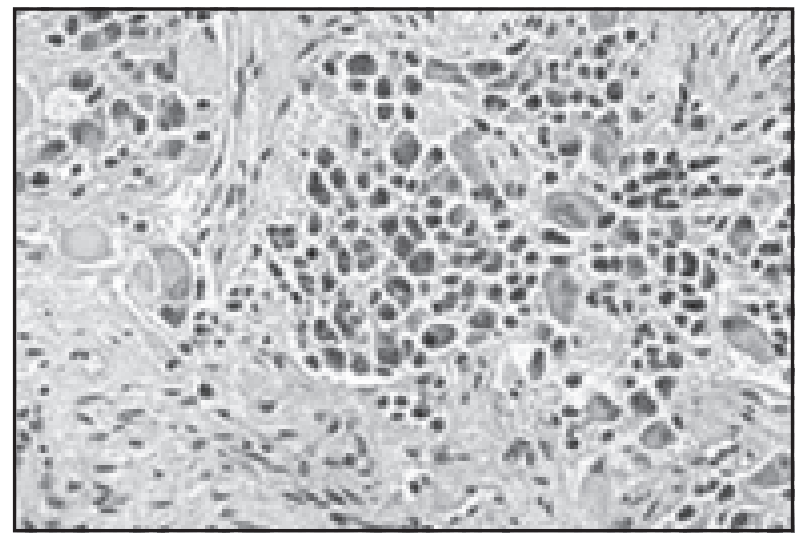

Microscopic view of neuroblastoma

On Immunohistochemistry neuron specific enolase(NSE) and chromogranin A were positive, thus leading to the diagnosis of Neuroblastoma. The young girl was thus referred to pediatric haemato oncology department of Dhaka medical college hospital for specific treatment.

\section{Discussion:}

As stated above, Neuroblastoma is an uncommon disease when it comes to over 5years aged children, especially in the neck and that too in girls. However, with proper and prompt clinical evaluation alongside relevant investigations, the diagnosis and subsequent treatment of such disorders is within limits, thus improving the mortality and morbidity associated with the disease.
However, we as treating doctors need to play an important role not only in the diagnosis \& treatment but also in providing psychological support to the "seemingly helpless" parents when they come to know about such a condition of their young ones. By explaining the disease, its prognosis after treatment, and addressing each part of it as it comes, would certainly pave a way for a bigger support than people expect from us.

\section{References:}

1. "eMedicine - Neuroblastoma : Article by Norman J Lacayo, MD". Retrieved 2008-07-30.

2. NHS Website

3. Janet Sassi, "Cellular Communication: Unraveling the Secrets of Histone Proteins", Fordham University, February 16, 2007

4. Bénard J, Raguénez G, Kauffmann A, et al. (October 2008). "MYCN-non-amplified metastatic neuroblastoma with good prognosis and spontaneous regression: a molecular portrait of stage 4S". Mol Oncol 2 (3): 261-71.doi:10.1016/ j.molonc.2008.07.002. PMID 19383347.

5. "ScienceDirect - The Lancet : Neuroblastoma".

6. "Neuroblastoma in children : Cancerbackup". Retrieved 2008-01-01.

7. Neuroblastoma: Pediatric Cancers: Merck Manual Professional". Retrieved 2008-01-01.

8. Friedman GK, Castleberry RP (December 2007). "Changing trends of research and treatment in infant neuroblastoma". Pediatr Blood Cancer 49(7 Suppl): 1060-5. doi:10.1002/ pbc.21354. PMID 17943963.

9. Rothenberg AB, Berdon WE, D’Angio GJ, Yamashiro DJ, Cowles RA (July 2009). "The association between neuroblastoma and opsoclonus-myoclonus syndrome: a historical review". Pediatr Radiol 39 (7): 7236.doi:10.1007/s00247-009-1282-x. ISBN [[Special:BookSources/2-470-09128-2|2-47009128-2]]. PMID 19430769.

10. Cheung, Nai-Kong (2005). Neuroblastoma. p. 66-67. Springer-Verlag.ISBN 3-540-40841-X.

11. Mossé YP, Laudenslager $M$, Longo $L$, et al. (October 2008). "Identification of ALK as a major familial neuroblastoma predisposition gene". 
Nature455 (7215): 930-5. doi:10.1038/ nature07261. PMC 2672043.PMID 18724359.

12. a b Howman-Giles R, Shaw PJ, Uren RF, Chung DK (2007)."Neuroblastoma and other neuroendocrine tumors". Semin Nucl Med 37(4): 286-302. doi:10.1053/j.semnuclmed. 2007. 02. 009.PMID 17544628.

13. $a b$ "Neuroblastoma Treatment - National Cancer Institute". Retrieved 2008-07-30.

14. Johnson E, Dean SM, Sondel PM (2007). "Antibody-based immunotherapy in high-risk neuroblastoma" (abstract). Expert Rev Mol Med 9 (34): 1-21.doi:10.1017/S1462399407000518. PMID 18081947.

15. Haase GM, Perez C, Atkinson JB (1999). "Current aspects of biology, risk assessment, and treatment of neuroblastoma". Semin Surg Oncol 16 (2): 91-104. doi:10.1002/(SICI)10982388(199903)16:2<91::AID-SSU3>3.0.CO;2-1. PMID 9988866.

16. Fish JD, Grupp SA (2007). "Stem cell transplantation for neuroblastoma". Bone Marrow Transplant 41 (2): 159.doi:10.1038/sj.bmt. 1705929. PMC 2892221. PMID 18037943.

17. Matthay KK, Villablanca JG, Seeger RC, et al. (1999). "Treatment of high-risk neuroblastoma with intensive chemotherapy, radiotherapy, autologous bone marrow transplantation, and 13cis-retinoic acid. Children's Cancer Group". N.
Engl. J. Med. 341 (16): 1165-73.doi:10.1056/ NEJM199910143411601. PMID 10519894.

18. $\mathrm{Yu} A L$, Gilman AL, Ozkaynak MF, et al. (September 2010). "Anti-GD2 antibody with GMCSF, interleukin-2, and isotretinoin for neuroblastoma". N. Engl. J. Med. 363 (14): 132434. doi:10.1056/NEJMoa0911123.PMID 20879881.

19. "Reduction From Seven to Five Cycles of Intensive Induction Chemotherapy in Children With High-Risk Neuroblastoma - Kushner et al. 22 (24): 4888 - Journal of Clinical Oncology".

20. $a b$ "Response and toxicity to a dose-intensive multi-agent chemotherapy induction regimen for high risk neuroblastoma (HR-NB): A Children's Oncology Group (COG A3973) study. - ASCO". Retrieved 2008-02-02.

21. Ceschel S, Casotto V, Valsecchi MG, et al. (Oct 2006). "Survival after relapse in children with solid tumors: a follow-up study from the Italian offtherapy registry". Pediatr Blood Cancer 47 (5): 560-6.doi:10.1002/pbc.20726. PMID 16395684.

22. Brodeur GM, Castleberry RP. Neuroblastoma. In: Pizzo PA, Poplack DG. Principles and practice of pediatric oncology, 3rd ed. 1997:761-797.

23. Franks LM, Bollen A, Seeger RC, Stram DO, Matthay KK (1997). "Neuroblastoma in adults and adolescents: an indolent course with poor survival". Cancer 79 (10): 2028-35. doi:10.1002/ (SICI)1097-0142(19970515)79:10<2028::AIDCNCR26>3.0.CO;2-V.PMID 9149032. 